\title{
In vivo function of Pgßglu-1 in the release of acetophenones in white spruce
}

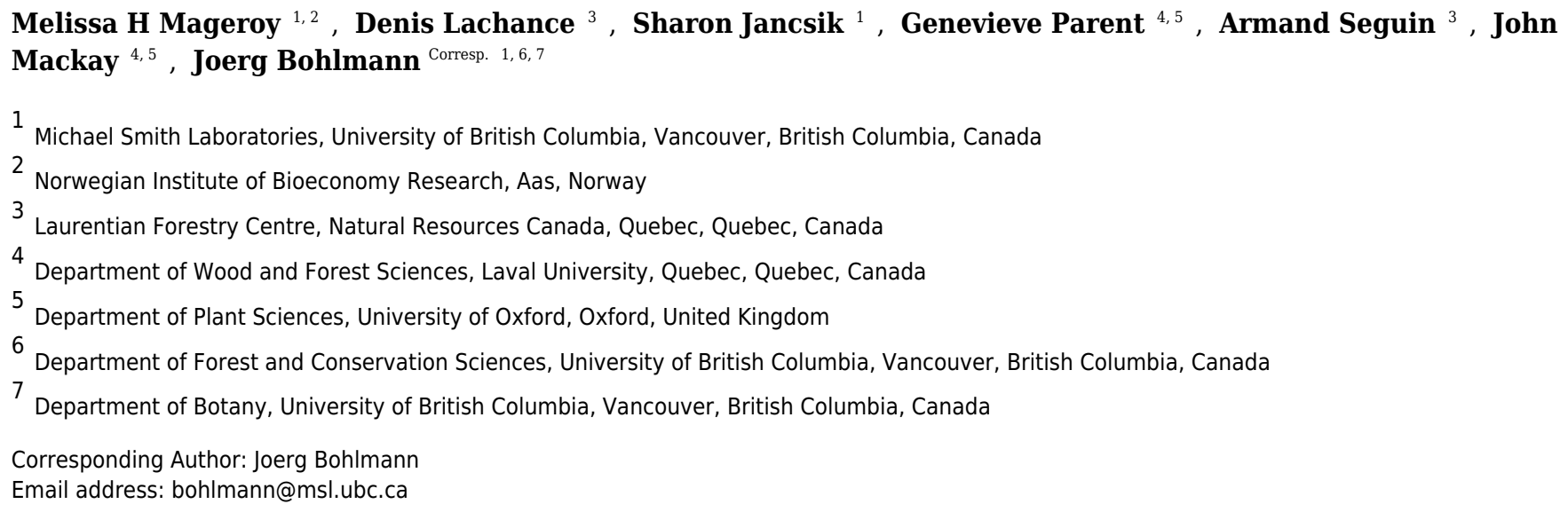

Eastern spruce budworm (Choristoneura fumiferiana Clemens) (ESBW) is a major forest pest which feeds on young shoots of white spruce (Picea glauca) and can cause landscape level economic and ecological losses. Release of acetophenone metabolites, piceol and pungenol, from their corresponding glycosides, picein and pungenin, can confer natural resistance of spruce to ESBW. A beta-glucosidase gene, Pgßglu-1, was recently discovered and the encoded enzyme was characterized in vitro to function in the release of the defensive acetophenone aglycons. Here we describe overexpression of Pgßglu-1 in a white spruce genotype whose metabolome contains the glucosylated acetophenones, but no detectable amounts of the aglycons. Transgenic overexpression of Pgßglu-1 resulted in release of the acetophenone aglycons in planta. This work provides in vivo evidence for the function of Pgßglu-1. 
In vivo function of $\mathrm{Pg} / \mathrm{glu}-1$ in the release of acetophenones in white spruce

Melissa H. Mageroy ${ }^{1,2}$, Denis Lachance ${ }^{3}$, Sharon Jancsik ${ }^{1}$, Geneviève Parent ${ }^{4,5}$, Armand Séguin $^{3}$, John Mackay ${ }^{4,5}$ and Joerg Bohlmann"1,6,7

${ }^{1}$ Michael Smith Laboratories, University of British Columbia, Vancouver, BC V6T 1Z4, Canada,

${ }^{2}$ Norwegian Institute of Bioeconomy Research, PO Box 115, N-1431 Ås, Norway

${ }^{3}$ Natural Resources Canada, Laurentian Forestry Centre, Québec, QC G1V 4C7, Canada,

${ }^{4}$ Department of Wood and Forest Sciences, Université Laval, Québec, QC G1V 0A6, Canada,

${ }^{5}$ Department of Plant Sciences, University of Oxford, South Parks Road, Oxford, OX1 3RB, UK

${ }^{6}$ Department of Forest and Conservation Sciences, University of British Columbia, Vancouver, BC V6T 1Z4, Canada

${ }^{7}$ Department of Botany, University of British Columbia, Vancouver, BC V6T 1Z4, Canada

Corresponding author:

Joerg Bohlmann

Email address: bohlmann@msl.ubc.ca 


\section{ABSTRACT}

2 Eastern spruce budworm (Choristoneura fumiferiana Clemens) (ESBW) is a major forest pest

3 which feeds on young shoots of white spruce (Picea glauca) and can cause landscape level

4 economic and ecological losses. Release of acetophenone metabolites, piceol and pungenol, from

5 their corresponding glycosides, picein and pungenin, can confer natural resistance of spruce to

6 ESBW. A beta-glucosidase gene, $P g \beta g l u-1$, was recently discovered and the encoded enzyme

7 was characterized in vitro to function in the release of the defensive acetophenone aglycons.

8 Here we describe overexpression of Pgßglu-1 in a white spruce genotype whose metabolome

9 contains the glucosylated acetophenones, but no detectable amounts of the aglycons. Transgenic

10 overexpression of Pgßglu- 1 resulted in release of the acetophenone aglycons in planta. This work provides in vivo evidence for the function of Pgßglu-1.

INTRODUCTION

Eastern spruce budworm (Choristoneura fumiferiana Clemens) (ESBW) is considered the most detrimental pest of spruce and fir forests in eastern North America. While populations of ESBW usually persist at endemic levels, outbreaks can last for years incurring landscape level ecological changes and major economic losses (Chang et al. 2015; MacLean 2016). With climate change, outbreaks of ESBW are predicted to increase in frequency and severity (Hennigar et al. 2013). Current forest management practices to control ESBW outbreak include costly aerial spraying of Bacillus thuringiensis (Bt) and the insect growth regulator Mimic ${ }^{(N R C A N ~ 2016) . ~}$ The Bt crylAb gene has been successfully overexpressed in white spruce and shown to be effective against ESBW (Lachance et al. 2007), however commercial deployment of transgenic trees is not permitted in Canada.

Recently, natural resistance to ESBW was discovered in white spruce (Picea glauca). Resistant genotypes accumulated the acetophenone aglycons piceol and pungenol as well as the corresponding glucosides picein and pungenin. Non-resistant genotypes only accumulated the acetophenone glucosides (Delvas et al. 2011). Parent et al. (2017) showed that the aglycons piceol and pungenol are the active defense compounds that contribute to resistance. We also showed that gene expression of Pgßglu- 1 was positively correlated with resistance, and in in 
vitro assays the encoded PgßGLU-1 enzyme cleaved the acetophenone glucosides, picein and pungenin, producing the biologically active aglycons (Mageroy et al. 2015). However, function of PgßGLU-1 has not yet been proven in planta and remained a critical but elusive part of the proof of function.

Here we report the successful overexpression of $P g \beta g l u-1$ in a white spruce genotype Pg653. While wildtype Pg653 plants do not accumulate detectable amounts of the acetophenone aglycons, overexpression of $P g \beta g l u-1$ resulted in the in planta formation of piceol and pungenol.

\section{METHODS}

Vector construction, Agrobacterium transformation and plant regeneration of PgBglu-1 overexpression white spruce

The full-length cDNA of Pgßglu-1 (GenBank KJ780719) or a modified green fluorescence protein ( $g f p)$ (Cambia) coding sequence were first cloned using the Gateway System (Invitrogen) into vector pMJM, containing the maize (Zea mays) ubiquitin promoter and the $35 \mathrm{~S}$ terminator (Levée et al. 2009), then digested with SbfI and sub-cloned into the binary vector pCAMBIA2300 (Supplemental Fig. 1; Supplemental Fig. 2). The resulting constructs were transformed into Agrobacterium tumefaciens strain C58 pMP90 (Hellens et al. 2000). Agrobacterium transformation of white spruce somatic embryonal masses (line Pg653) and subsequent selection and growth of transformants was performed as described by Klimaszewska et al. (2001). Kanamycin resistance was used as the selection marker. Somatic embryo maturation, germination, acclimatization and transfer of somatic seedlings to soil were performed according to Klimaszewska et al. (2004).

\section{LRE-qPCR of embryogenic tissue and somatic seedlings}

Linear regression of efficiency (LRE) qPCR (Rutledge 2011) was used to confirm transformation and to measure and compare absolute transcript abundance levels in both embryogenic tissue and somatic seedlings of 11 selected Pgßglu-1 and $11 \mathrm{gfp}$ transformed lines. RNA was isolated from up to $100 \mathrm{mg}$ fresh weight of embryogenic tissue or from the pooled epicotyls of two 2-months old somatic seedlings using the RNeasy Plant mini kit (Qiagen) with on-column RNase-Free DNase (Qiagen) treatment. Primers were designed as previously described (Foster et al. 2015) 
62

63

64

65

66

67

68

69

with primers PgßGLU1-f-5'- GCCATAAGGGAGGGAGCAG; PgßGLU1-r-5'CTCGCCCACTCAAAGCCGT or GFP-f-5'- GCCCGACAACCACTACCTGA; GFP-r-5'GCGGTCACGAACTCCAGCAG used to analyze the $g \beta g l u$ - 1 and $g f p$ lines respectively. cDNA synthesis, primer design, and PCR thermocycling conditions were conducted as described by Foster et al. (2015) with the exceptions that a two-step amplification protocol of 45 cycles was used with a 120 second annealing/elongation step at $65^{\circ} \mathrm{C}$. Gene expression was normalized using the two white spruce reference genes YLS8 and EF1 $\alpha$ (Rutledge et al. 2013). Transcript abundance quantification was performed using a Java program based on linear regression of efficiency previously described (Rutledge 2011).

\section{Plant growth conditions}

After growth on germination media for three months, somatic seedlings were planted into cones (Figure 1b) and maintained in a greenhouse under natural light and growth lights (16 h; 600W HPS). Temperatures were set with a low of $19^{\circ} \mathrm{C}$ and, within the limitations of a greenhouse that is not fully temperature controlled, to a high of $23.5^{\circ} \mathrm{C}$. Plants were allowed to grow for eight months and then placed at $4^{\circ} \mathrm{C}$ with minimal light for two weeks to induce flushing. Plants were placed on the benchtop at $22^{\circ} \mathrm{C}$ for one week to transition from the cold and then moved into a growth chamber with $16 \mathrm{~h}$ light at $22^{\circ} \mathrm{C}$ and $8 \mathrm{~h}$ dark at $16^{\circ} \mathrm{C}$.

\section{RT-qPCR of plants grown for 6 months}

Total RNA was isolated from needles of plants grown for six months using PureLink ${ }^{\circledR}$ Plant RNA Reagent (ThermoFisher) using approximately $100 \mathrm{mg}$ tissue according to manufacturer's instructions. RNA integrity and concentration was measured using Bioanalyzer 2100 RNA Nano chip assays (Agilent) following the manufacturer's protocol. Equal RNA amounts were used for cDNA synthesis with the iScript Reverse Transcription Supermix (Bio-Rad). qRT-PCR reactions were performed on a Bio-Rad CFX96 Real-time system using the SsoFast kit (Bio-Rad) in triplicate. Relative transcript abundance was calculated using efficiency corrected $\Delta C_{\mathrm{T}}$ and $\Delta \Delta C_{\mathrm{T}}$ values based on ELF-1 $\alpha$ as the reference gene. Target-specific oligonucleotides were as follows: ELF-1 $\alpha-f-5$ '-CCCTTCCTCACTCCAACTGCATA; ELF-1 $\alpha-\mathrm{r}-5$ 'TCGGCGGTGGCAGAGTTTACATTA; or PgßGLU1-f_-5'-TTGGATCCTCTGAAGGTGT; 
92 PgßGLU1-r-5'-TCCCTCCCTTATGGCTTC. Target specificity was confirmed by sequence

93 verification of representative amplicons.

94

95

96

97

98

100

101

102

103

104

105

106

107

108

109

110

111

112

113

114

115

116

117

118

119

120

121

122

\section{Metabolite analysis}

For the time course study of acetophenone glucoside deglycosylation, tissue was ground and left on the bench top for $4 \mathrm{~h}, 8 \mathrm{~h}$, and $24 \mathrm{~h}$ before adding extraction solvent [100\% methanol containing $1 \mathrm{mg} / \mathrm{ml}$ benzoic acid as the internal standard]. For all other metabolite extractions, $100 \mathrm{mg}$ of tissue was placed in a vial and $1 \mathrm{~mL}$ of extraction solvent was immediately added. The vial was capped and placed at $4{ }^{\circ} \mathrm{C}$ with shaking overnight. The supernatant was removed and placed in a new vial. For liquid chromatography-mass spectrometry (LC-MS) analysis, samples were diluted 1:10 by diluting $100 \mu \mathrm{L}$ of supernatant in $900 \mu \mathrm{L}$ of $100 \%$ methanol. LCMS analysis was performed using a LC-MSD-Trap-XCT_plus with a SB-C18, 15-cm column (Agilent). An injection volume of $10 \mu \mathrm{L}$ was used. Solvent A was water with $0.2 \%(\mathrm{v} / \mathrm{v})$ formic acid; solvent B was 100\% (v/v) acetonitrile with $0.2 \%(\mathrm{v} / \mathrm{v})$ formic acid. The following gradient was used: increase to $5 \%$ solvent B from 0 to $0.5 \mathrm{~min}$; increase to $22 \%$ solvent B from 0.5 to 5.0 min; increase to $35 \%$ solvent B from 5.0 to $10.0 \mathrm{~min}$; increase to $50 \%$ solvent B from 10.0 to $13.0 \mathrm{~min}$; increase to $95 \%$ solvent B from 13.0 to $16.0 \mathrm{~min}$; holding $95 \%$ solvent B from 16.0 to $17.0 \mathrm{~min}$; decrease to $5 \%$ solvent $\mathrm{B}$ from 17.0 to $17.1 \mathrm{~min}$. Column flow rate was $0.8 \mathrm{~mL} \mathrm{~min}^{-1}$. Piceol and picein were identified using the extracted ion 135(-), the parent mass (-1) of piceol. Pungenol and pungenin were identified using the extracted ion 151(-), the parent mass (-1) of pungenol.

\section{RESULTS AND DISCUSSION}

\section{Overexpression of SBW defense gene Pgßglu-1}

We overexpressed the cDNA of Pgßglu-1 in planta to validate the function of this gene and its encoded enzyme activity in the release of acetophenone aglycons from the corresponding glucosides in white spruce foliage (Figure 1). We used the white spruce genotype Pg653 to test the effect of overexpression of Pg/glu-1 for two reasons: 1) Pg653 is a well-established somatic embryogenic line for white spruce transformations. 2) This line shows a metabolite phenotype that contains the acetophenone glucosides picein and pungenin, which are the proposed in vivo substrates for Pgßglu-1 enzyme activity, but contains minimal detectable amounts of the 
123

124

125

126

127

corresponding aglycons piceol and pungenol. Thus, Pg653 provides a suitable background for de novo formation of piceol and pungenol in transgenic plants. Agrobacterium transformation of the coding region of PgBglu-1 driven under maize (Zea mays) ubiquitin promoter was used to produce transgenic white spruce lines. A $g f p$ reporter gene was overexpressed in white spruce as a control.

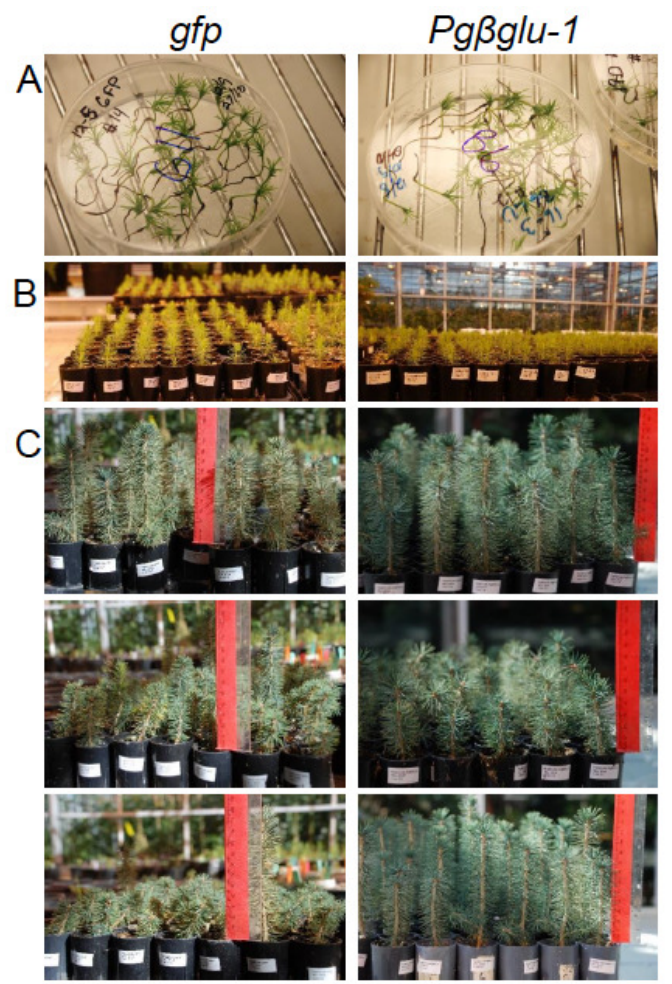

Figure 1. Transgenic white spruce seedlings. A, C, E, G, and I show control white spruce seedlings expressing gfp. $\mathrm{B}, \mathrm{D}, \mathrm{F}, \mathrm{H}$, and $\mathrm{J}$ show white spruce seedlings overexpressing Pgßglu-1. (A-B) Transgenic white spruce somatic seedlings were grown on selective media for three months. (B-C) Plantlets were then transferred into cones and placed in the greenhouse. (D-J) After six months of growth, on average, the Pgßglu-1 overexpressing seedlings appeared healthier and taller.

\section{Evaluation of possible negative effects of transgene overexpression}

Levels of transgene gene expression were evaluated in both embryonal tissue and somatic seedlings using qPCR (Figure 2). Overall transcript levels of the $g f p$ transgene were higher in both sample types compared to the $P g \beta g l u-1$ transgene. The lower levels of $P g \beta g l u-1$ transcripts could indicate some phytotoxic effects, as toxic compounds are often glycosylated in plants for self-protection. However, $P g \beta g l u-1$ overexpressing young plants appeared to be healthier than $g f p$ expressing plants (Figure 1c) under greenhouse conditions, including naturally occurring 
136 biotic and abiotic stresses. Although previous studies have shown $g f p$ to be non-toxic in plants

137 (Millwood et al. 2010; Tian et al. 1999), deleterious effects have been noted in mammalian cells

138 (Liu et al 1999). The observed plant growth difference may be due to physiological adaptations

139 to cope with effects that may arise from high levels of $g f p$ expression (Steward 2001). For

140 example, under stress conditions, which increase the production of free radicals, plant cells may 141 not be able to compensate as well for high expression of $g f p$ leading to negative growth effects.

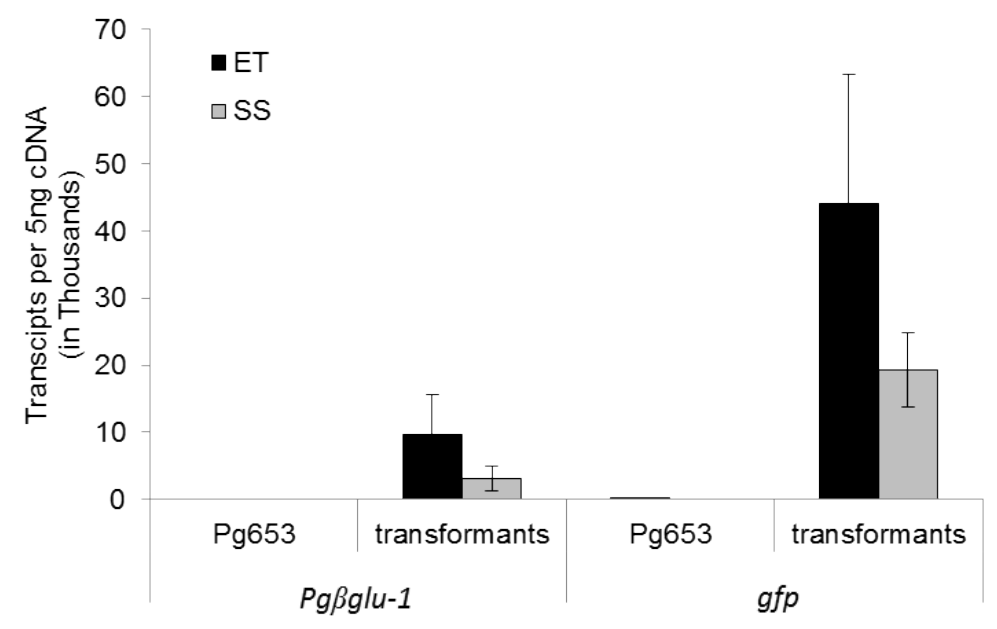

Figure 2. LRE-qPCR quantification of transgene expression in embryonic tissue (ET) and somatic seedlings (SS) of transformed lines. Transcript abundance was calculated as the number of transcripts in $5 \mathrm{ng}$ of synthesized cDNA. Pg653 represent the untransformed control line. Overall, higher expression of $g f p$ was observed in ET and SS than $P g \beta g l u-1$. Error bars represent standard deviation. $\mathrm{N}=11$

Young seedlings overexpressing Pg/glu-1 do not accumulate aceotophenone aglycons

No significant levels of acetophenone aglycons were observed in either $g f p$ controls or Pg/glu- 1 overexpressing transgenic seedlings after the first six months of growing in the greenhouse. To test if we could observe aglycon production in $P g \beta g l u-1$ overexpressing foliage when tissues were disrupted, we chose one high, one medium, and one low Pg $\beta g l u-1$ expressing line based on transcript abundance in six-month old seedlings (Figure 3a). Tissue was ground and the disrupted tissue left at room temperature for up to $24 \mathrm{~h}$ before metabolite extraction. In this time course test, we observed much greater release of the acetophenone aglycons piceol and pungenol in $P g \beta g l u-1$ overexpressing lines compared to $g f p$ controls (Figure $3 b$ ). In nature, acetophenone aglycons are produced in resistant white spruce foliage without tissue disturbance (Mageroy et al. 2015). The requirement of tissue disruption to produce the aglycons in the Pgßglu-1 
154 overexpressing lines may indicate that PgßGLU-1 protein is prevented from interacting with 155 glucosides in the young seedlings, perhaps due to differential localization of the enzyme and the 156 substrate or some reversible inhibition or inactivation of the enzyme. As Pg $\beta g l u-1$ was expressed 157 under a constitutive promoter it is plausible that this expression may be spatially and temporally 158 amiss or that reversible protein modification rendered it inactive in young seedlings. However, 159 the ability of $P g \beta g l u-1$ overexpressing lines to produce greater amounts of aglycon when tissue 160 was disturbed provided additional proof for the function of this gene and its encoded protein. It 161 is possible that this increased accumulation of acetophenone aglycons upon tissue distruption 162 may contribute enhanced resistance in plants overexpressing $P g \beta g l u-1$. This remains to be tested 163 in future work.
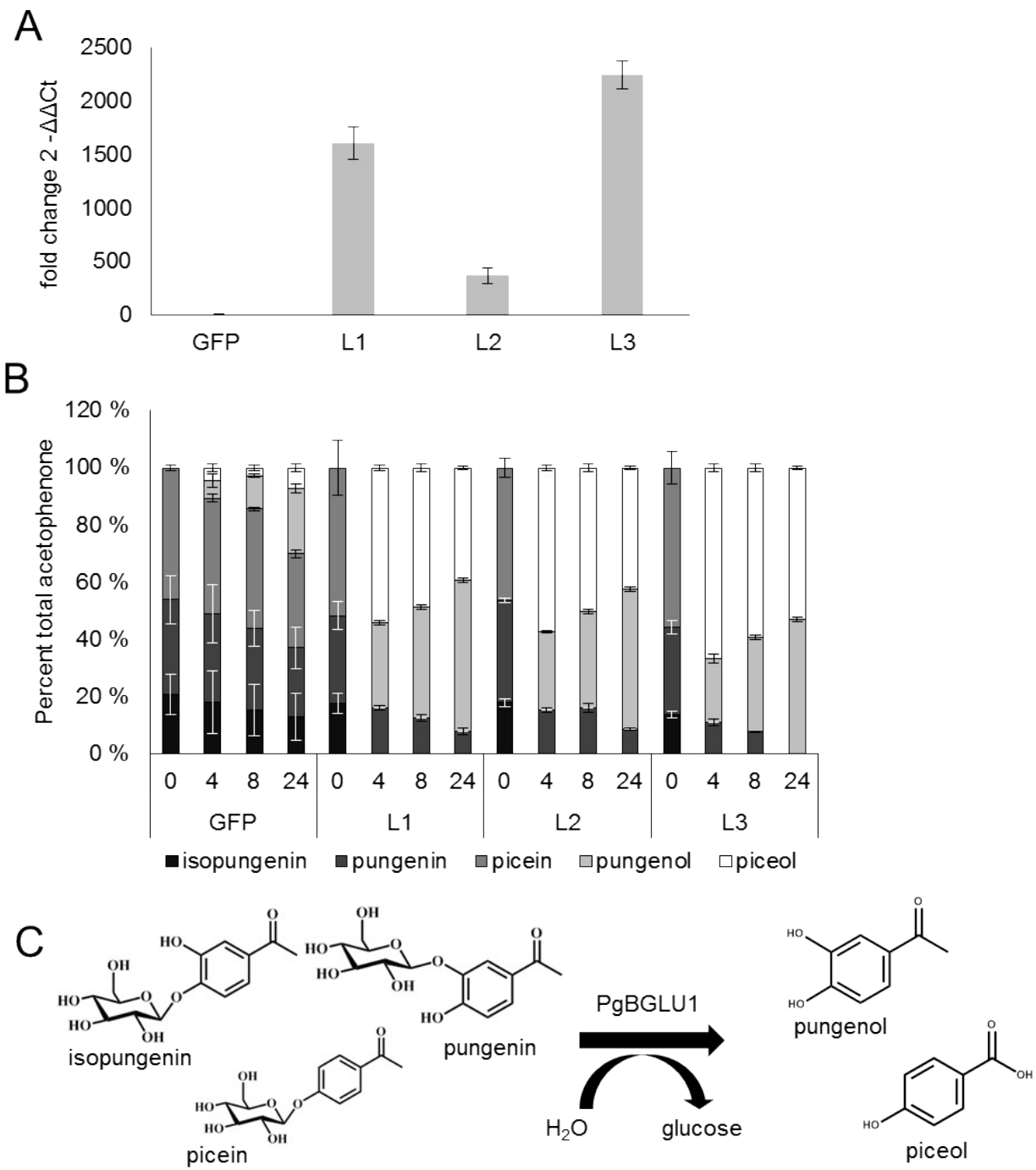

Figure 3. Analysis of transgene expression and $\beta$-glucosidase potential in 6-months old transgenic white spruce seedlings. (A) After 6 months of growth in the greenhouse, qRT-PCR 
was used to determine the fold change in $P g \beta g l u-1$ expression between $g f p$ expressing plants and three independent lines of $P g \beta g l u-1$ overexpressing plants. Representative low, medium and high $P g \beta g l u-1$ expressing lines were chosen for comparative metabolite analysis. GFP represents the average of four independent lines. Error bars represent standard deviation. $N=3$ (B) To test if acetophenone glucoside could be released in $P g \beta g l u$ - 1 overexpressing seedlings, needles were grounds and the disrupted tissue left for $0 \mathrm{~h}, 4 \mathrm{~h}, 8 \mathrm{~h}$, and $24 \mathrm{~h}$ before extracting metabolites. A much larger proportion of acetophenone aglycons was released in $P g \beta g l u-1$ overexpressing trees compared very small proportion of acetophenone aglycons released in $g f p$ overexpressing tissue. Error bars represent standard error. $\mathrm{N}=3$. (C) The structures of glucosylated acetophenone and their aglycons with the catalytic function of PgßGLU-1.

\section{Seedlings overexpressing $P g \beta g l u-1$ accumulate acetophenone aglycons in newly growing} shoot tissue after induced dormancy

Since white spruce is a perennial tree species, acetophenone production may be influenced by plant development beyond the first growth phase. We tested this possibility by carrying eightmonth old seedlings through a simulated complete growth cycle including bud set, winter dormancy, and new bud flush, which involved a cold treatment in the dark and subsequent return to normal light and temperature conditions favorable to active vegetative growth. Bud flush began three weeks after returning trees to normal growth conditions. Following a gap period of no detectable levels of acetophenone glucosides and aglycons in the newly flushing shoots, accumulation of both acetophenone glucosides and the corresponding aglycons was detected at eight weeks after the beginning of new shoot growth (Figure 4a). While both the acetophenone glucosides and aglycons were observed in the $P g \beta g l u$ - 1 overexpressing seedlings, no substantial quantities of the aglycons were detected in the $g f p$ transgenic control seedlings. Under these in planta conditions, overexpression of $P g \beta g l u-1$ led to higher amounts of the picein-derived aglycon piceol compared to the pungenol aglycon (Figure 4b). Accumulation of piceol also correlated with the difference of Pgßglu-1 transcript levels in low, medium and high expressing lines (Figure 3a and Figure 4b). These results conclusively confirm in planta function of $P g \beta g l u$ - $l$ in the release of acetophenone aglycons and their accumulation in intact plant tissue. In previous work, we reported the in vitro kinetic parameters of the PgßGLU-1 enzyme with picein as the substrate, but not for pungenin as this substrate is not a commercially available (Mageroy et al. 2015). The present results suggest that Pg $\beta G L U-1$ is more active on picein, compared to pungenin, in planta. 



Figure 4. Altered acetophenone glucoside and aglycon profiles in new shoots of white spruce seedlings after bud flush. Eight weeks after the beginning of bud flush, acetophenone aglycons were detected in extraction from intact $P g \beta g l u-1$ overexpressing shoots. (A) The extracted ion chromatogram (EIC) for the parent mass of piceol (-135). (B) The EIC for the parent mass of pungenol (-151). (C) Piceol and pungenol were quantified using authentic standards. A higher amount of piceol was released in Pgßglu-1 overexpressing shoots compared to the amount of pungenol. Error bars represent standard error. $\mathrm{N}=3$. CONCLUSIONS

We showed that overexpressing $P g \beta g l u-1$ in a white spruce genotype that does not naturally contain acetophenone aglycons leads to the in planta formation of the resistance metabolite piceol, and in disrupted tissues also the additional formation of pungenol. The results validate previously reported in vitro function of Pg/glu- 1 and its encoded PgßGLU-1 enzyme. The different results obtained with young seedling before bud flush and seedlings that had passed through bud set and new bud flush point out the need for caution when evaluating phenotypes of young seedling overexpressing a transgene. As we found, the altered metabolite phenotype was 
195

196

197

198

199

200

201

202

203

204

205

206

207

208

209

210

211

212

213

214

215

216

217

218

219

220

221

222

223

224

225

not observable in intact tissue until after the first bud flush. Conditions of spruce metabolism that provide the precursors for altered metabolism may vary depending on the developmental stage of seedlings, where precursors for defense metabolism may only become fully accessible after the seedlings have gone through an initial growth phase or a dormancy phase. In future work, effects of the $P g \beta g l u-1$ transgene expression and altered acetophenone profiles in transgenic Pg653 trees will be tested with insect feeding test, which will require production and maturation of a larger number of young trees.

\section{ACKNOWLEDGMENTS}

We thank Lina Madilao for her assistance with LCMS. JB is a UBC Distinguished University Scholar.

\section{REFERENCES}

Chang W-Y., Lantz VA., Hennigar CR., MacLean DA. 2012. Economic impacts of forest pests: a case study of spruce budworm outbreaks and control in New Brunswick, Canada. Canadian Journal of Forest Research 42:490-505. DOI: 10.1139/x11-190.

Delvas N., Bauce É., Labbé C., Ollevier T., Bélanger R. 2011. Phenolic compounds that confer resistance to spruce budworm. Entomologia Experimentalis et Applicata 141:35-44. DOI: 10.1111/j.1570-7458.2011.01161.x.

Foster AJ., Pelletier G., Tanguay P., Séguin A. 2015. Transcriptome Analysis of Poplar during Leaf Spot Infection with Sphaerulina spp. PloS one 10:e0138162. DOI: 10.1371/journal.pone.0138162.

Hellens R., Mullineaux P., Klee H. 2000. A guide to Agrobacterium binary Ti vectors. Trends in Plant Science 5:446-451. DOI: 10.1016/S1360-1385(00)01740-4.

Hennigar CR., Erdle TA., Gullison JJ., Maclean DA. 2013. Re-examining wood supply in light of future spruce budworm outbreaks: A case study in New Brunswick. Forestry Chronicle 89:42-53. DOI: 10.5558/tfc2013-010.

Hjältén J., Axelsson EP. 2015. GM trees with increased resistance to herbivores: trait efficiency and their potential to promote tree growth. Frontiers in plant science 6:279. DOI: 10.3389/fpls.2015.00279.

Klimaszewska K., Lachance D., Pelletier G., Lelu M-AL., Séguin A. 2001. Regeneration of Transgenic Picea Glauca, P. Mariana, and P. Abies After Cocultivation of Embryogenic 
Tissue With Agrobacterium tumefaciens. In Vitro Cellular \& Developmental Biology Plant 37:748-755. DOI: 10.1079/IVP2001230.

Klimaszewska K., Rutledge RG., Séguin A. 2004. Genetic transformation of conifers utilizing somatic embryogenesis. In: Peña L ed. Transgenic Plants: Methods and Protocols. Totowa, NJ, United States: Humana Press, 151-64.

Lachance D., Hamel LP., Pelletier F., Valero J., Bernier-Cardou M., Chapman K., van Frankenhuyzen K., Séguin A. 2007. Expression of a Bacillus thuringiensis cry1 Ab gene in transgenic white spruce and its efficacy against the spruce budworm (Choristoneura fumiferana). Tree Genetics \& Genomes 3:153-167. DOI: 10.1007/s11295-006-0072-y.

Levée V., Major I., Levasseur C., Tremblay L., MacKay J., Séguin A. 2009. Expression profiling and functional analysis of Populus WRKY23 reveals a regulatory role in defense. New Phytologist 184:48-70. DOI: 10.1111/j.1469-8137.2009.02955.x.

Liu H-S., Jan M-S., Chou C-K., Chen P-H., Ke N-J. 1999. Is Green Fluorescent Protein Toxic to the Living Cells? Biochemical and Biophysical Research Communications 260:712-717. DOI: http://dx.doi.org/10.1006/bbrc.1999.0954.

MacLean DA. 2016. Impacts of insect outbreaks on tree mortality, productivity, and stand development. The Canadian Entomologist 148:S138-S159. DOI: 10.4039/tce.2015.24.

Mageroy MH., Parents G., Germanos G., Giguère I., Delvas N., Maaroufi H., Bauce E., Bohlmann J., Mackay JJ. 2015. Expression of the b-glucosidase gene Pg/glu- 1 underpins natural resistance of white spruce against spruce budworm. The Plant Journal 81:68-80. DOI: $10.1111 /$ tpj.12699.

Millwood RJ., Moon HS., Stewart Jr. CN. 2010. Fluorescent Proteins in Transgenic Plants. In: Geddes CD ed. Reviews in Fluorescence 2008. Springer New York, 387-403. DOI: 10.1007/978-0-387-88722-7.

NRCAN. 2016.Spruce budworm. Available at http://www.nrcan.gc.ca/forests/fire-insectsdisturbances/top-insects/13383 (accessed February 24, 2016).

Parent GJ., Giguère I., Germanos G., Lamara M., Bauce É., MacKay JJ. 2017. Insect herbivory (Choristoneura fumiferana, Tortricidea) underlies tree population structure (Picea glauca, Pinaceae). Scientific Reports, Published online: 16 February 2017; | doi:10.1038/srep42273 199:379-398. DOI: 10.1038/SREP42273.

Rutledge RG., Stewart D. 2010. Assessing the performance capabilities of LRE-based assays for absolute quantitative real-time PCR. PLOS ONE 5. DOI: 10.1371/journal.pone.0009731.

Rutledge RG. 2011. A Java program for LRE-based real-time qPCR that enables large-scale absolute quantification. PloS one 6:e17636. DOI: 10.1371/journal.pone.0017636. 
260

261

262

263

264 265

266

267

Rutledge RG., Stewart D., Caron S., Overton C., Boyle B., MacKay J., Kilmaszewska K. 2013. Potential link between biotic defense activation and recalcitrance to induction of somatic embryogenesis in shoot primordial from adult trees of white spruce (Picea glauca). BMC Plant Biology 13:116. DOI: 10.1186/1471-2229-13-116

Stewart CNJ. 2001. The utility of green fluorescent protein in transgenic plants. Plant Cell Reports 20:376-382.

Tian LN., Levée V., Mentag R., Charest PJ., Séguin A. 1999. Green fluorescent protein as a tool for monitoring transgene expression in forest tree species. Tree Physiology 19:541-546. 